\title{
LOCAL SURGERY AND APPLICATIONS TO THE THEORY OF QUADRATIC FORMS
}

\author{
BY WILLIAM PARDON
}

Communicated July 25, 1975

Let $A$ be a unitary ring with involution and let $L_{n}^{h}(A)$ denote the surgery obstruction group in dimension $n$, defined by C. T. C. Wall in Chapters 5 and 6 of [13], for surgery to a homotopy equivalence. In this note a new local surgery theory [8], [9] is used to produce a localization sequence for Wall's $L^{h}$-groups (cf. Example 2 following Theorem 1). This sequence, together with a MayerVietoris sequence derived from Sharpe's unitary periodicity for $K U_{i}, 0 \leqslant i \leqslant 2$, can be used to make computations which include and extend many of the results of Bak [1], Bass [2], Karoubi [6], and Wall [14]. The approach we outline to the determination of surgery obstructions should be more accessible to topologists than other recent treatments, because the analysis involves only two nontrivial but essentially geometric tools: the localization sequence and the Mayer-Vietoris sequence. The first can be realized geometrically in the case of group rings; the second involves a formal construction in algebraic $K$-theory, together with Sharpe's study of the unitary Steinberg group, which can also be realized geometrically [11].

The localization sequence. Let $A$ be a ring with involution and $\bar{A}$ a classical ring of quotients with respect to a multiplicative subset $\Sigma \subseteq A$. Five terms (starting with $\left.L_{2 k+1}^{h}(A)\right)$ of the following localization sequence have been obtained by Karoubi [6] independently.

THEOREM 1. Let $A$ be a ring with involution, $\bar{A}$ a ring of quotients as above. Then there exists a sequence of groups $L_{n}^{t}(A, \Sigma)$ and a long exact sequence

$$
\cdots \rightarrow L_{n+1}^{h}(\bar{A}) \rightarrow L_{n}^{t}(A, \Sigma) \rightarrow L_{n}^{h}(A) \rightarrow L_{n}^{h}(\bar{A}) \rightarrow L_{n-1}^{t}(A, \Sigma) \rightarrow \cdots
$$

ExAmples. Let $\bar{A}$ denote the injective hull of $A$. In the examples below, $\bar{A}$ is a classical ring of quotients.

(1) If $D$ is a Dedekind domain, $\bar{D}=F$ its fraction field, and the involution is trivial, then Theorem 1 (modified to replace $L^{h}$-groups with $U$-groups of [10, $\S 3])$ gives the Milnor exact sequence of [5],

$$
0 \rightarrow W(D) \rightarrow W(F) \rightarrow \coprod_{p} w(D / p),
$$

AMS (MOS) subject classifications (1970). Primary 13D15, 15A63, 57D65; Secondary $18 \mathrm{~F} 25,57 \mathrm{C} 45$. 
where $W(A)$ is the Witt group of $A[5]$. In particular, this part of the sequence answers a question raised by A. Durfee: the obstruction to lifting a form on a $D$-torsion module back to $W(F)$ lies in the group $U_{4 k-1}(D)$. (Durfee shows the obstruction group is $C / C^{2}$, where $C$ is the class group of $D$, when $D$ is the ring of integers in a number field; in this case $U_{4 k-1}(D)=C / C^{2}$.) More generally $D$ may be an order in the semisimple algebra $\bar{D}$.

(2) “Local Surgery". If $\pi$ is finite then $\bar{Z} \pi=\mathbf{Q} \pi$. In this example $L_{n}^{t}\left(\mathbf{Z} \pi, \mathbf{Z}^{x}\right)\left(=L_{n}^{t}(\mathbf{Z} \pi)\right.$ in [8], [9]) is a surgery obstruction group, Theorem 1 is a formal consequence of Chapter 9 of [13], and the geometric analysis in [8] provides the method of proof in Theorem 1. $L_{n}^{t}\left(Z \pi, Z^{x}\right)$ is the obstruction group for completing surgery on a normal map $f: M \rightarrow X$ such that $f$ is a rational homology equivalence, where we require that the bordisms of $f$ be rationally homology-equivalent to $X$. Using the techniques of [13], modified for surgery on $\mathbf{Z}_{n}$-manifolds instead of spheres, we obtain the following algebraic description. Let $I$ be the category of $\mathbf{Z} \pi$-modules $S$ admitting a short free $\mathbf{Z} \pi$-resolution, together with nondegenerate $\epsilon$-hermitian linking and self-linking forms, $b$ and $q$, as in Theorem 5.2 of [12]. Then the groups $L_{n}^{t}(\mathrm{Z} \pi)$ are the $L$-groups in the category $I$ in the style of $[10]$.

(3) If $A=\mathbf{Z Z}$ (viewed as the ring of finite Laurent series) and $\Sigma=$ $\{p(t) \in \mathbf{Z Z} \mid p(1)= \pm 1\}$, then $L_{n+2}^{t}(A, \Sigma)$ is the knot group $C_{n}$.

This yields a new algebraic description of $C_{n}$. (A much more general result on codimension two imbeddings has been obtained by Justin Smith.)

(4) By an elementary calculation (i.e. without using unitary stability theorems or the solution of the congruence subgroup problem as in [7]) one may show $L_{3}\left(Z Z Z_{p}\right)=0$ by proving that $L_{3}^{t}\left(Z Z_{p}\right)=L_{3}^{t}(Z \zeta)+L_{3}^{t}(Z)$ and applying Theorem $1(Z \zeta=$ cyclotomic integers). Here is a sample application.

THEOREM 2 [1]. $L_{n}^{h}(\mathrm{Z} \pi)=0$ if $|\pi|$ and $n$ are odd.

The idea of the proof is to reduce to the case of odd order cyclic groups using [3]. Then from Sharpe's unitary periodicity theorem we derive a MayerVietoris sequence for cartesian squares satisfying certain conditions. Squares satisfying the conditions appear in [4] for cyclic $p$-groups, $p$ odd. One may tensor these squares over $\mathbf{Z}$ with $\mathbf{Z} \pi^{\prime}$, where $\pi^{\prime}$ is cyclic, $\left(\left|\pi^{\prime}\right|, p\right)=1$, and retain the conditions. Thus we reduce to the case of rings of integers, which we can treat with Theorem 1.

AdDED IN PROof. A sequel to [6] has appeared (Ann. Sci. Ecole Norm Sup., t.8, fasc. 1, 1975) in which Karoubi obtains more of Theorem 1, assuming $1 / 2 \in A$.

\section{BIBLIOGRAPHY}

1. A. Bak, Odd dimension surgery groups of odd order groups vanish (preprint).

2. H. Bass, $L_{3}$ finite abelian groups, Ann. of Math. (2) 99 (1974), 118-153. MR 50 \#497. 
3. A. Dress, Induction and structure theorems for Grothendieck and Witt rings of orthogonal representations of finite groups, Bull. Amer. Math. Soc. 79 (1973), 741-745. MR 49 \#7345.

4. S. Galovich, The class group of a cyclic p-group, J. Algebra 30 (1974), 368-387.

5. D. Husemoller and J. Milnor, Symmetric bilinear forms, Ergebnisse der Mathematik und ihrer Grenzgebiete, Band 73, Springer-Verlag, Berlin and New York, 1973.

6. M. Karoubi, Localisation des formes quadratiques (preprint).

7. R. Lee, Computation of Wall groups, Topology 10 (1974), 149-176.

8. W. Pardon, The exact sequence of a localization in L-theory, Thesis, Princeton Univ., 1974.

9. The exact sequence of a localization in L-theory, Notices Amer. Math. Soc. 22 (1975), A-477-A-478. Abstract \#75T-G43.

10. A. Rainicki, Foundations of algebraic L-theory. I, Proc. London Math. Soc. 27 (1973), $101-125$.

11. R. Sharpe, Surgery and unitary $K_{2}$, Lecture Notes in Math., vol. 343, SpringerVerlag, Berlin and New York, 1973.

12. C. T. C. Wall, Surgery of non-simply-connected manifolds, Ann. of Math. (2) 84 (1966), 217-276. MR 35 \#3692.

13. - Surgery on compact manifolds, Academic Press, New York and London, 1970.

14. The classification of Hermitian forms. I-IV, Compositio Math. 22 (1970), 425-451; Invent. Math. 18 (1972), 119-141; Invent. Math. 19 (1973), 59-71. MR 43 \#7425; 48 \#2184; \#2185.

DEPARTMENT OF MATHEMATICS, COLUMBIA UNIVERSITY, NEW YORK, NEW YORK 10027 York 10027

Current address: Department of Mathematics, Columbia University, New York, New 